\title{
Photoproduction of meson and baryon resonances in a chiral unitary approach
}

\author{
E. Oset ${ }^{1}$, E. Marco ${ }^{2}$, J.C. Nacher ${ }^{1}$, J.A. Oller ${ }^{3}$, J.R. Peláez ${ }^{4}$, A. Ramos ${ }^{5}$, H. Toki ${ }^{6}$ \\ ${ }^{1}$ Departamento de Física Teórica and IFIC, Universidad de Valencia, Burjassot(Valencia), Spain \\ 2 Physik-Department, Technische Universität München, D-85747 Garching, Germany \\ ${ }^{3}$ Forschungzentrum Jülich, IKP(Th), D-52425 Jülich, Germany. \\ ${ }^{4}$ Departamento de Física Teórica. Universidad Complutense de Madrid, 28040 Madrid, Spain \\ ${ }^{5}$ Department d'Estructura i Constituents de la Matéria, Universitat de Barcelona, Barcelona, Spain \\ ${ }^{6}$ Research Center for Nuclear Physics (RCNP), Osaka University, Ibaraki, Osaka 567-0047, Japan
}

\begin{abstract}
By means of a coupled channel non-perturbative unitary approach, it is possible to extend the strong constrains of Chiral Perturbation Theory to higher energies. In particular, it is possible to reproduce the lowest lying resonances in mesonmeson scattering up to $1.2 \mathrm{GeV}$ using the parameters of the $O\left(p^{2}\right)$ and $O\left(p^{4}\right)$ Chiral Lagrangian. The meson baryon sector can also be tackled along similar lines. We report on an update of these results showing some examples of photon induced reactions where the techniques have been recently applied.
\end{abstract}

\section{Introduction}

Chiral Perturbation Theory $(\chi P T)$ [] has proved very successful in order to describe the physics of mesons at very low energies. The key point of the whole approach is to identify the lightest pseudoscalar mesons $\pi, K$ and $\eta$ as the Goldstone bosons associated to the chiral symmetry breaking. These particles will be the only degrees of freedom at low energies and their interactions can be described in terms of the most general effective Lagrangian which respects the chiral symmetry constraints.

So far as this is a low energy approach, the amplitude of a given process is basically given as an expansion in the external momenta over the scale of symmetry breaking $4 \pi f_{\pi} \simeq 1.2 \mathrm{GeV}$. The approach is known to provide a good description of meson interactions up to about $500 \mathrm{MeV}$. However, if one is interested in resonances in particular, as it happens in meson spectroscopy, there is little that one can do with just plain $\chi P T$. The method that we expose here naturally leads to low lying resonances and allows one to face many problems so far intractable within $\chi P T$.

The method incorporates the following elements: 1) Unitarity is implemented exactly; 2) It can deal with coupled channels allowed with pairs of particles from the lightest octets of pseudoscalar mesons and $\left(\frac{1}{2}^{+}\right)$baryons; 3) A chiral expansion in powers of the external four-momentum of the lightest pseudoscalars is done for $\operatorname{Re} T^{-1}$, instead of the $T$ matrix itself as it is done in standard $\chi P T$. 
We sketch here the steps involved in this expansion for the meson meson interaction. One starts from a $K$ matrix approach in coupled channels where unitarity is automatically fulfilled and writes

$$
T^{-1}=K^{-1}-i \sigma
$$

where $T$ is the scattering matrix, $K$ is a real matrix in the physical region and $\sigma$ is a diagonal matrix which measures the phase-space available for the intermediate states

$$
\sigma_{n n}(s)=-\frac{k_{n}}{8 \pi \sqrt{s}} \theta\left(s-\left(m_{1 n}+m_{2 n}\right)^{2}\right)
$$

where $k_{n}$ is the on shell CM momentum of the meson in the intermediate state $n$ and $m_{1 n}, m_{2 n}$ are the masses of the two mesons in the state $n$. The meson meson states considered here are $K \bar{K}, \pi \pi$, $\pi \eta, \eta \eta, \pi K, \pi \bar{K}, \eta K, \eta \bar{K}$. Since $K$ is real in the physical region, from eq. (1) one sees that $K^{-1}=$ Re $T^{-1}$. In non-relativistic Quantum Mechanics, in the scattering of a particle from a potential, it is possible to expand $K^{-1}$ in powers of the momentum of the particle at low energies as follows (in the s-wave for simplicity)

$$
\operatorname{Re} T^{-1} \equiv K^{-1}=\sigma \cdot \operatorname{ctg} \delta \propto-\frac{1}{a}+\frac{1}{2} r_{0} k^{2}
$$

with $k$ the particle momentum, $a$ the scattering length and $r_{0}$ the effective range.

The ordinary $\chi \mathrm{PT}$ expansion up to $O\left(p^{4}\right)$ is given by [1]

$$
T=T_{2}+T_{4}
$$

where $T_{2}$ is obtained from the lowest order chiral Lagrangian, $L^{(2)}$, and is of $O\left(p^{2}\right)$, whereas $T_{4}$ contains one loop diagrams in the s, t, u channels, constructed from the lowest order Lagrangian, tadpoles and the finite contribution from the tree level diagrams of the $L^{(4)}$ Lagrangian and is $O\left(p^{4}\right)$. This last contribution, after a suitable renormalization, is just a polynomial, $T^{(p)}$. Our $T^{-1}$ matrix, starting from eq. (4) is given by

$$
\begin{aligned}
T^{-1} & =\left[T_{2}+T_{4}+\ldots\right]^{-1}=T_{2}^{-1}\left[1+T_{4} T_{2}^{-1}+\ldots\right]^{-1} \\
& =T_{2}^{-1}\left[1-T_{4} T_{2}^{-1}+\ldots\right]=T_{2}^{-1}\left[T_{2}-T_{4}\right] T_{2}^{-1}
\end{aligned}
$$

Due to the fact that $\operatorname{Im} T_{4}=T_{2} \sigma T_{2}$, the above equation is nothing but eq. (1), but using eq. (4) to expand $K^{-1}=\operatorname{Re} T^{-1}$. Inverting the former result, one obtains:

$$
T=T_{2}\left[T_{2}-T_{4}\right]^{-1} T_{2}
$$

which is the coupled channel generalization of the inverse amplitude method of [2].

Once this point is reached one has several options to proceed:

a) A full calculation of $T_{4}$ within the same renormalization scheme as in $\chi P T$ can be done. The eight $L_{i}$ coefficients from $L^{(4)}$ are then fitted to the existing meson meson data on phase shifts and 
inelasticities up to $1.2 \mathrm{GeV}$, where 4 meson states are still unimportant. This procedure has been carried out in [2, 3]. The resulting $L_{i}$ parameters are compatible with those used in $\chi P T$. At low energies the $O\left(p^{4}\right)$ expansion for $T$ of eq. (6) is identical to that in $\chi P T$. However, at higher energies the nonperturbative structure of eq. (6), which implements unitarity exactly, allows one to extend the information contained in the chiral Lagrangians to much higher energy than in ordinary $\chi$ PT. Indeed it reproduces the resonances present in the $\mathrm{L}=0,1$ partial waves.

b) A technically simpler and equally successful additional approximation is generated by ignoring the crossed channel loops and tadpoles and reabsorbing them in the $L_{i}$ coefficients given the weak structure of these terms in the physical region. The fit to the data with the new $\hat{L}_{i}$ coefficients reproduces the whole meson meson sector, with the position, widths and partial decay widths of the $f_{0}(980), a_{0}(980), \kappa(900), \rho(770), K^{*}(900)$ resonances in good agreement with experiment [4]. A cut off regularization is used in [4 for the loops in the s-channel. By taking the loop function with two intermediate mesons

$$
G_{n n}(s)=i \int \frac{d^{4} q}{(2 \pi)^{4}} \frac{1}{q^{2}-m_{1 n}^{2}+i \epsilon} \frac{1}{(P-q)^{2}-m_{2 n}^{2}+i \epsilon},
$$

where $P$ is the total meson meson momentum, one immediately notices that

$$
\operatorname{Im} G_{n n}(s)=\sigma_{n n}
$$

Hence, we can write

$$
\operatorname{Re} T_{4}=T_{2} \operatorname{Re} G T_{2}+T_{4}^{(p)}
$$

where $\operatorname{Re} G$ depends on the cut off chosen for $|\vec{q}|$. This means that the $\hat{L}_{i}$ coefficients of $T_{4}^{(p)}$ depend on the cut off choice, much as the $L_{i}$ coefficients in $\chi P T$ depend upon the regularization scale.

c) For the $\mathrm{L}=0$ sector (also in $\mathrm{L}=0, \mathrm{~S}=-1$ in the meson baryon interaction) a further technical simplification is possible. In these cases it is possible to choose the cut off such that, given the relation between $\operatorname{Re} G$ and $T_{4}^{(p)}$, this latter term is very well approximated by $\operatorname{Re} T_{4}=T_{2} \operatorname{Re} G T_{2}$. This is possible in those cases because of the predominant role played by the unitarization of the lowest order $\chi P T$ amplitude, which by itself leads to the low lying resonances, and because other genuine QCD resonances appear at higher energies.

In such a case eq. (5) becomes

$$
T=T_{2}\left[T_{2}-T_{2} G T_{2}\right]^{-1} T_{2}=\left[1-T_{2} G\right]^{-1} T_{2}
$$

or, equivalently,

$$
T=T_{2}+T_{2} G T
$$

which is a Bethe-Salpeter equation with $T_{2}$ and $T$ factorized on shell outside the loop integral, with $T_{2}$ playing the role of the potential. This option has proved to be successful in the $\mathrm{L}=0$ meson meson sector in [5] and in the $\mathrm{L}=0, \mathrm{~S}=-1$ meson baryon sector in [6]. 
In the meson baryon sector with $\mathrm{S}=0$, given the disparity of the masses in the coupled channels $\pi N, \eta N, K \Sigma, K \Lambda$, the simple "one cut off approach" is not possible. In [7] higher order Lagrangians are introduced while in [8] different subtraction constants in G are incorporated in each of the former channels leading in both cases to acceptable solutions when compared with the data.

In fig. 1 we show the results done with the method of [4] for some selected phase shifts and inelasticities in the meson meson sector, showing resonances in different channels, (see [9] for an update of the results). The agreement with the meson meson data is quite good up to $1.2 \mathrm{GeV}$ and the parameters $\hat{L}_{i}$ obtained from the fit are essentially compatible with those of $\chi P T$.
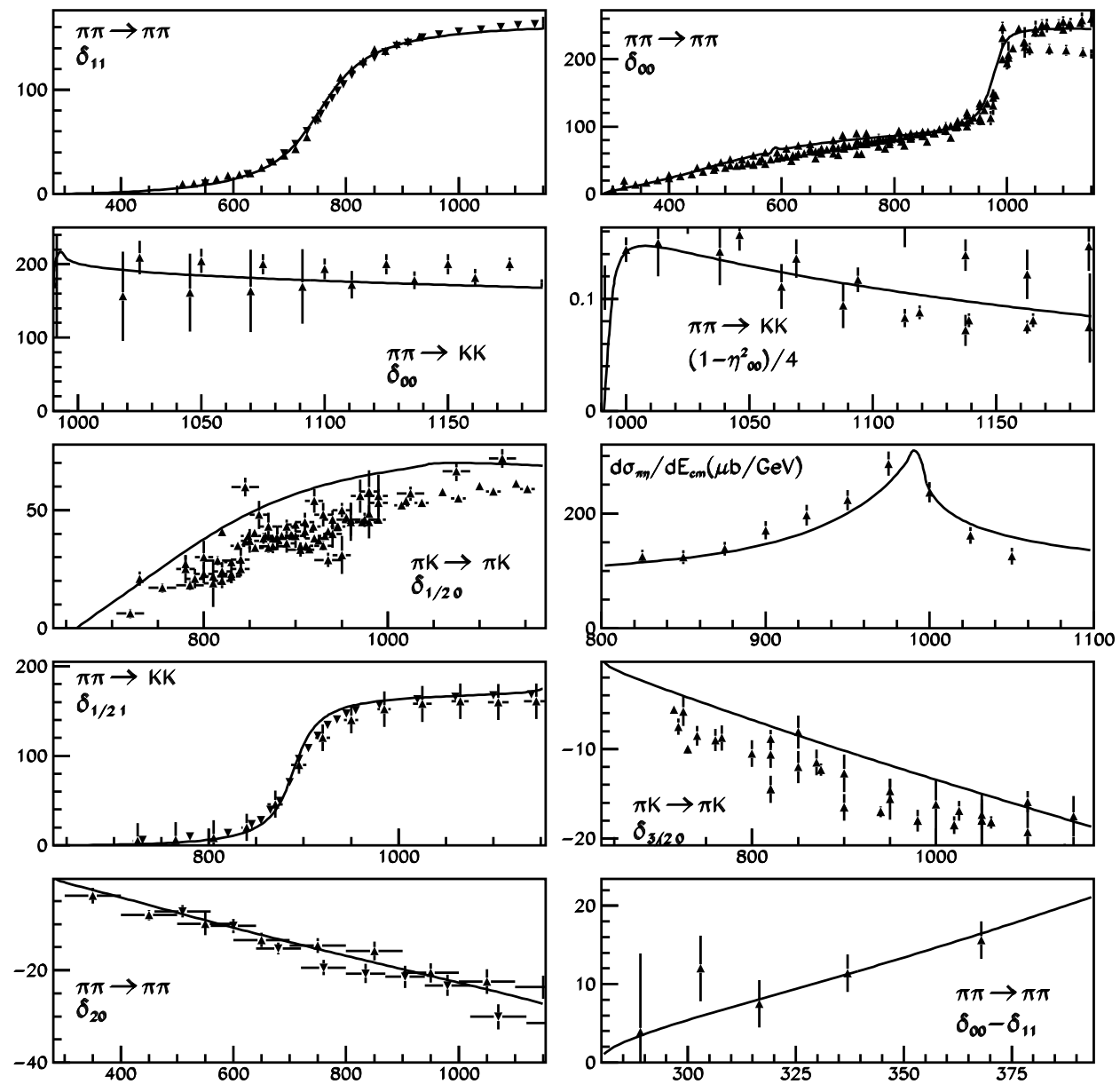

Figure 1: Meson-meson scattering results of the non-perturbative chiral approach. For the data see references in [2, 4, 3, 18]. Horizontal scale in $\mathrm{MeV}$.

\section{$2 \quad \bar{K} N$ interaction in free space}

The meson-baryon interaction Lagrangian at lowest order in momentum is given by

$$
L_{1}^{(B)}=\left\langle\bar{B} i \gamma^{\mu} \frac{1}{4 f^{2}}\left[\left(\Phi \partial_{\mu} \Phi-\partial_{\mu} \Phi \Phi\right) B-B\left(\Phi \partial_{\mu} \Phi-\partial_{\mu} \Phi \Phi\right)\right]\right\rangle
$$

where $\Phi$ represents the octet of pseudoscalar mesons and $B$ the octet of $1 / 2^{+}$baryons. The symbol \langle\rangle denotes the trace of $\mathrm{SU}(3)$ matrices. 
The coupled channel formalism requires to evaluate the transition amplitudes between the different meson-baryon channels. For $K^{-} p$ scattering there are ten channels, namely $K^{-} p, \bar{K}^{0} n, \pi^{0} \Lambda$, $\pi^{0} \Sigma^{0}, \pi^{+} \Sigma^{-}, \pi^{-} \Sigma^{+}, \eta \Lambda, \eta \Sigma^{0}, K^{+} \Xi^{-}$and $K^{0} \Xi^{0}$, while in the case of $K^{-} n$ scattering there are six: $K^{-} n, \pi^{0} \Sigma^{-}, \pi^{-} \Sigma^{0}, \pi^{-} \Lambda, \eta \Sigma^{-}$and $K^{0} \Xi^{-}$. These amplitudes have the form

$$
V_{i j}=-C_{i j} \frac{1}{4 f^{2}} \bar{u}\left(p_{i}\right) \gamma^{\mu} u\left(p_{j}\right)\left(k_{i \mu}+k_{j \mu}\right),
$$

where $p_{j}, p_{i}\left(k_{j}, k_{i}\right)$ are the initial, final momenta of the baryons (mesons) and $C_{i j}$ are $\mathrm{SU}(3)$ coefficients that can be found in Ref. [6]. At low energies the spatial components can be neglected and the amplitudes reduce to

$$
V_{i j}=-C_{i j} \frac{1}{4 f^{2}}\left(k_{j}^{0}+k_{i}^{0}\right)
$$

The coupled-channel BS equations in the center of mass frame read

$$
T_{i j}=V_{i j}+\overline{V_{i l} G_{l} T_{l j}}
$$

where the indices $i, l, j$ run over all possible channels and $\overline{V_{i l} G_{l} T_{l j}}$ corresponds to the loop integral involving $V, T$ and the meson baryon propagators of $G$, all functions of the loop variable. However, as was shown in Ref. [6], the off-shell part of $V_{i l}$ and $T_{l j}$ goes into renormalization of coupling constants and $V_{i l}, T_{l j}$ factorize outside the integral with their on-shell values, thus reducing the problem to one of inverting a set of algebraic equations.

Table 1: $K^{-} p$ threshold ratios and $K^{-} N$ scattering lengths

\begin{tabular}{|l|c|c|}
\hline$\gamma=\frac{\Gamma\left(K^{-} p \rightarrow \pi^{+} \Sigma^{-}\right)}{\Gamma\left(K^{-} p \rightarrow \pi^{-} \Sigma^{+}\right)}$ & This work & Exp. \\
\hline$R_{c}=\frac{\Gamma\left(K^{-} p \rightarrow \text { charged }\right)}{\Gamma\left(K^{-} p \rightarrow \text { all }\right)}$ & 0.627 & $2.36 \pm 0.04$ [10] \\
\hline$R_{n}=\frac{\Gamma\left(K^{-} p \rightarrow \pi^{0} \Lambda\right)}{\Gamma\left(K^{-} p \rightarrow \text { neutral }\right)}$ & 0.213 & $0.664 \pm 0.011$ [10 \\
\hline$a_{K^{-} p}(\mathrm{fm})$ & $-1.00+\mathrm{i} 0.94$ & $-0.67+\mathrm{i} 0.64[11]$ \\
& & $-0.98($ from $\operatorname{Re}(a))$ \\
& & $(-0.78 \pm 0.18)+\mathrm{i}(0.49 \pm 0.37)$ \\
\hline$a_{K^{-} n}(\mathrm{fm})$ & $0.53+\mathrm{i} 0.62$ & $0.37+\mathrm{i} 0.60$ [1] \\
\hline
\end{tabular}

The value of the cut-off, $q_{\max }=630 \mathrm{MeV}$, was chosen to reproduce the $K^{-} p$ scattering branching ratios at threshold, while the weak decay constant, $f=1.15 f_{\pi}$, was taken in between the pion and 
kaon ones to optimize the position of the $\Lambda(1405)$ resonance. The predictions of the model for several scattering observables are summarized in Table 1. Cross sections for $K^{-} p$ scattering to different channels are also calculated in [6] and good results are obtained for low energies of the kaons where the s-wave is dominant.

A recent application of these methods in the $\mathrm{S}=0$ sector is done in [13], where the $\Delta(1232)$ resonance is also nicely reproduced.

\section{Application to the photoproduction of meson baryon pairs in resonant states}

As quoted above, a good description of the interaction of $K^{-} p$ and its coupled channels is obtained in terms of the lowest order Lagrangians and the Bethe Salpeter equation with a single cut off. One of the interesting features of the approach is the dynamical generation of the $\Lambda(1405)$ resonance just below the $K^{-} p$ threshold. The threshold behavior of the $K^{-} p$ amplitude is thus very much tied to the properties of this resonance. Modifications of these properties in a nuclear medium can substantially alter the $K^{-} p$ and $K^{-}$nucleus interaction and experiments looking for these properties are most welcome. Some electromagnetic reactions appear well suited for these studies. Application of the chiral unitary approach to the $K^{-} p \rightarrow \gamma \Lambda, \gamma \Sigma^{0}$ reactions at threshold has been carried out in [14] and a fair agreement with experiment is found. In particular one sees there that the coupled channels are essential to get a good description of the data, increasing the $K^{-} p \rightarrow \gamma \Sigma^{0}$ rate by about a factor 16 with respect to the Born approximation.

In a recent paper 15 the $\gamma p \rightarrow K^{+} \Lambda(1405)$ reaction was proposed as a means to study the properties of the resonance, together with the $\gamma A \rightarrow K^{+} \Lambda(1405) A^{\prime}$ reaction to see the modification of its properties in nuclei. The resonance $\Lambda(1405)$ is seen in its decay products in the $\pi \Sigma$ channel, but as shown in 15 the sum of the cross sections for $\pi^{0} \Sigma^{0}, \pi^{+} \Sigma^{-}, \pi^{-} \Sigma^{+}$production has the shape of the resonance $\Lambda(1405)$ in the $\mathrm{I}=0$ channel. Hence, the detection of the $K^{+}$in the elementary reaction, looking at $d \sigma / d M_{I}\left(M_{I}\right.$ the invariant mass of the meson baryon system which can be induced from the $K^{+}$momentum), is sufficient to get a clear $\Lambda(1045)$ signal. In nuclear targets Fermi motion blurs this simple procedure (just detecting the $K^{+}$), but the resonance properties can be reconstructed by observing the decay products in the $\pi \Sigma$ channel. In fig. 2 we show the cross sections predicted for the $\gamma p \rightarrow K^{+} \Lambda(1405)$ reaction looking at $K^{+} \pi^{0} \Sigma^{0}, K^{+}$all and $K^{+} \Lambda(1405)$ (alone). All of them have approximately the same shape and strength given the fact that the $\mathrm{I}=1$ contribution is rather small. In the figure the dashed dotted line indicates what one should expect to see in nuclei, just detecting the $K^{+}$, from the effect of Fermi motion. The energy chosen for the photon is $E_{\gamma}=1.7 \mathrm{GeV}$ which makes it suitable of experimentation at SPring8/RCNP, where the experiment is planned [16], and 


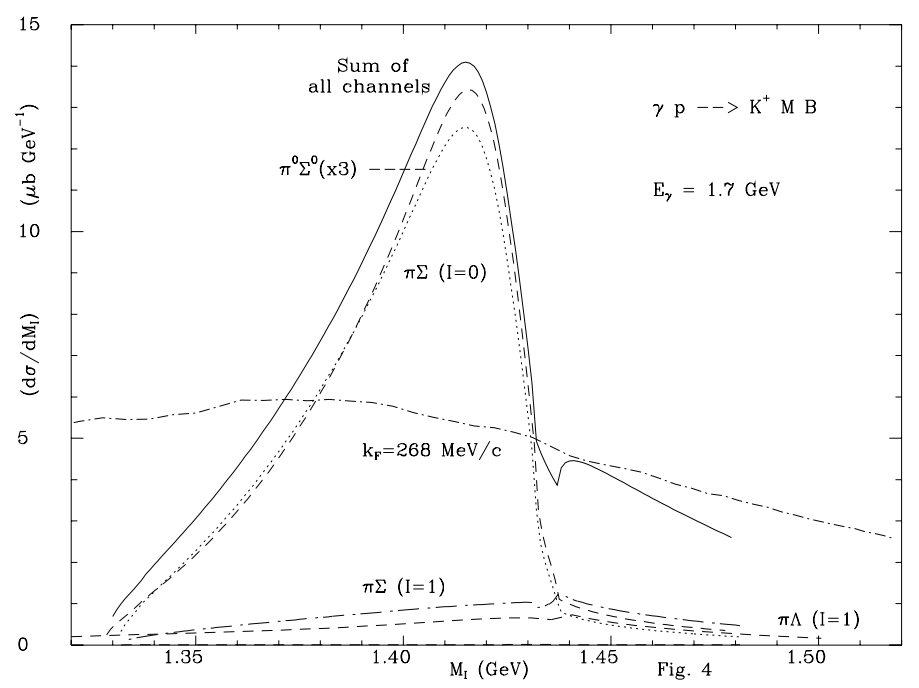

Figure 2: Cross section for $\gamma p \rightarrow K^{+} X$ with $X=$ all, $\pi^{0} \Sigma^{0}, \Lambda(1405)$.

\section{TJNAF.}

One variant of this reaction is the crossed channel reaction $K^{-} p \rightarrow \Lambda(1405) \gamma$. This reaction, for a $K^{-}$momentum in the 300 to $500 \mathrm{MeV} / \mathrm{c}$ range, shows clearly the $\Lambda(1405)$ resonant production [17] and has the advantage that the analogous reaction in nuclei still allows the observation of the $\Lambda(1405)$ resonance with the mere detection of the photon, the Fermi motion effects being far more moderate than in the case of the $\gamma A \rightarrow K^{+} \Lambda(1405) X$ reaction which requires larger photon momenta and induces a broad distribution of $M_{I}$ for a given $K^{+}$momentum.

\section{Photoproduction of resonant two meson states}

Another application which can be done using the same reaction is the photoproduction of resonant two meson states. Particularly the $f_{0}(980)$ and $a_{0}(980)$ resonances. These states appear in $L=0$ in isospin zero and one respectively. The scalar sector of the meson is very controversial and the chiral unitary theory has brought a new perspective on these states. In particular, it has been possible to identify the lightest scalar octet, made of the $\sigma, f_{0}(980), a_{0}(980)$ and $\kappa(900)$ resonances. All of them can be simply generated by unitarization of the lowest order ChPT, with just a cutoff as a free parameter.

The $O\left(p^{4}\right)$ chiral parameters can be understood as the residual contact terms that appear when one integrates out heavier states and the resulting Lagrangian is that of ChPT. Hence, the values of the chiral constants can be related to the masses and widths of the preexisting heavier resonances ("Resonance Saturation Hypothesis"). Indeed, most of their values are saturated by vector resonances alone (that is vector meson dominance) but some other parameters still need the existence of scalar states. Recently [18], using the N/D unitarization method with explicit resonances added to the lowest order ChPT Lagrangian, it has been established that these heavier scalar states should appear with a mass around $1.3-1.4 \mathrm{GeV}$ for the octet and $1 \mathrm{GeV}$ for the singlet. In addition, the $\sigma, \kappa, a_{0}$ and a 
strong contribution to the $f_{0}$, were also generated from the unitarization of the ChPT lowest order. These states still survive when the heavier scalars are removed. That agrees with our observation that the $\sigma, \kappa, f_{0}$ and $a_{0}$ are generated independently of the chiral parameters, that is, of the preexisting scalar nonet, which is heavier. In addition, it was also stablished in that work that the physical $f_{0}(980)$ resonance is a mixture between the discussed strong $K \bar{K}$ scattering contribution and the preexisting singlet resonance with a mass around $1 \mathrm{GeV}$. Since Chiral Perturbation Theory does not deal with quarks and gluons, it is very hard to make any conclusive statement about the nature of these states ( $q \bar{q}$, four-quark, molecule, etc...), unless we make additional assumptions. However, any model of the nature of these states should be able to explain the different features of these resonances as they appear in the chiral unitary approach.

In addition, it would be very interesting to obtain further information from other processes. In the present case the reaction suggested is [19] $\gamma p \rightarrow p M$. where $M$ is either of the resonances $a_{0}(980)$ or $f(980)$. In practice the meson $\mathrm{M}$ will decay into two mesons,$\pi \pi$ or $K \bar{K}$ in the case of the $f_{0}(980)$ or $K \bar{K}, \pi \eta$ in the case of the $a_{0}(980)$.

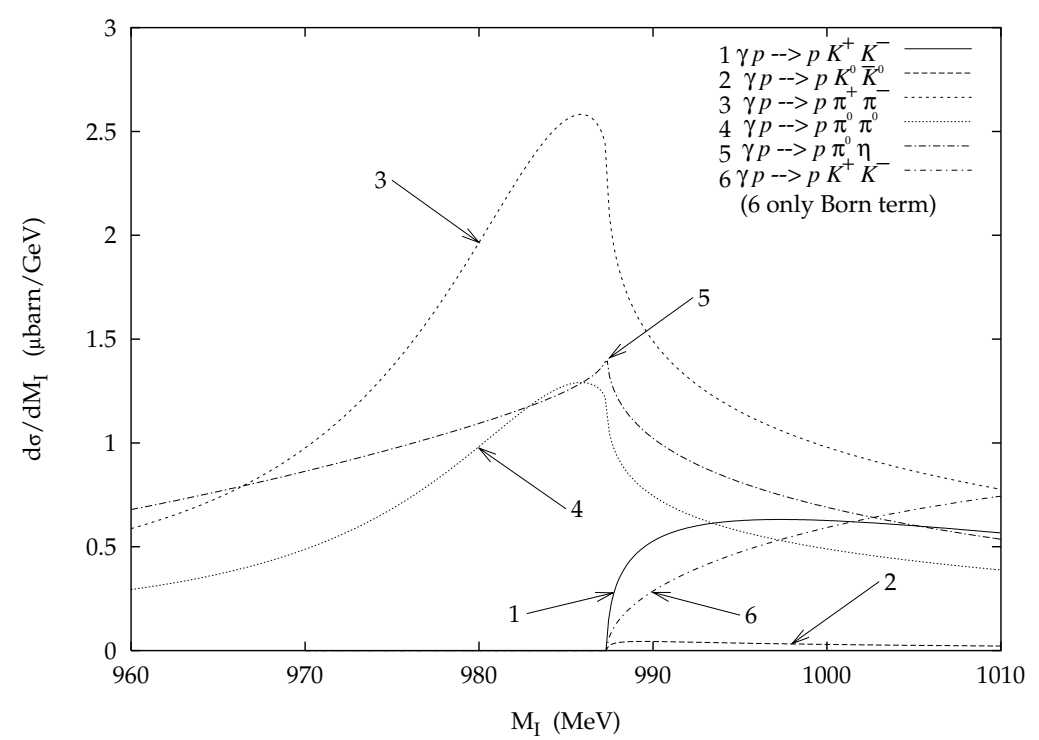

Figure 3: Results for the photoproduction cross section on protons as a function of the invariant mass of the meson-meson system.

In Fig. 3 we show the results for the 5 channels considered. We observe clear peaks for $\pi^{+} \pi^{-}$, $\pi^{0} \pi^{0}$ and $\pi^{0} \eta$ production around $980 \mathrm{MeV}$. The peaks in $\pi^{+} \pi^{-}$and $\pi^{0} \pi^{0}$ clearly correspond to the formation of the $f_{0}(980)$ resonance, while the one in $\pi^{0} \eta$ corresponds to the formation of the $a_{0}(980)$. The $\pi^{0} \pi^{0}$ cross section is $\frac{1}{2}$ of the $\pi^{+} \pi^{-}$one due to the symmetry factor. The $K^{+} K^{-}$and $K^{0} \bar{K}^{0}$ production cross section appears at energies higher than that of the resonances and hence do not show the resonance structure. Yet, final state interaction is very important and increases appreciably the $K^{+} K^{-}$production cross section for values close to threshold with respect to the Born approximation.

It is interesting to notice that the $f_{0}(980)$ resonance shows up as a peak in the reaction. This is in contrast to the cross section for $\pi \pi \rightarrow \pi \pi$ in $I=0$ which exhibits a minimum at the $f_{0}$ energy 
because of the interference between the $f_{0}$ contribution and the $\sigma(500)$ broad resonance.

However, we should bear in mind that we have plotted there the contribution of the $f_{0}$ resonance alone. The tree level contact term and Bremsstrahlung diagrams, plus other contributions which would produce a background, are not considered there.

In any case it is interesting to quote in this respect that a related reaction from the dynamical point of view, which also involves the interaction of two mesons in the final state, the $\phi \rightarrow \pi^{0} \pi^{0} \gamma$ and the $\phi \rightarrow \pi^{0} \eta \gamma$ decay, which have been measured recently at Novosibirsk [20], show clearly the $f_{0}$ and $a_{0}$ excitation, respectively, in the invariant mass spectra of the two mesons. A theoretical study along the lines reported here has been done in 21] where a good description of the experimental spectra as well as the absolute rates is obtained.

\section{Summary}

We have reported on the unitary approach to meson meson and meson baryon interaction using chiral Lagrangians, which has proved to be an efficient method to extend the information contained in these Lagrangians to higher energies where $\chi P T$ cannot be used. This new approach has opened the doors to the investigation of many problems so far intractable with $\chi P T$ and a few examples have been reported here. We have applied these techniques to the problem of photoproduction of scalar mesons $f_{0}(980), a_{0}(980)$ and the photoproduction of the $\Lambda(1405)$, a resonant state of meson baryon in the $S=-1$ sector and have found signals which are well within measuring range in present facilities. The experimental implementation of these experiments confronted with the theoretical predictions will contribute with new tests of these emerging pictures implementing chiral symmetry and unitarity, which for the moment represent the most practical approach to QCD at low energies.

\section{Acknowledgments.}

This work is partly supported by DGICYT, contract number PB 96-0753.

\section{References}

[1] S. Weinberg, Physica A96 (1979) 327; J Gasser annd H. Leutwyler, Ann. Phys. NY 158 (1984) 142; J. Gasser and H. Leutwyler, Nucl. Phys. B250 (1985) 465,517,539.

[2] T. N. Truong, Phys. Rev. Lett. 66 (1988) 2526; Phys. Rev. Lett. 67 (1991) 2260; A. Dobado, M.J.Herrero and T.N. Truong, Phys. Lett. B235 (1990) 134 ; A. Dobado and J.R. Peláez, Phys. Rev. D47 4883 (1993)4883 ; Phys. Rev. D56 (1997) 3057. 
[3] F. Guerrero and J. A. Oller, Nucl. Phys. B 537(1999)459

[4] J.A. Oller, E. Oset and J.R. Peláez, Phys. Rev. Lett. 80 (1998) 3452; Phys. Rev. D59 (1999) 074001.

[5] J. A. Oller and E. Oset, Nucl. Phys. A620(1997)438; erratum Nucl. Phys. A624(1999)407

[6] E. Oset and A. Ramos, Nucl. Phys. A635(1998)99

[7] N. Kaiser, T. Waas and W. Weise, Nucl. Phys. A612 (1997) 297

[8] J.C. Nacher, A. Parreño, E. Oset, A. Ramos, A.Hosaka and M. Oka, nucl-th/9906018

[9] J.A. Oller, E. Oset and J.R. Peláez, Preprint.

[10] D.N. Tovee et al., Nucl. Phys. B33(1971)493. R.J. Nowak et al., Nucl. Phys. B139 (1978)61.

[11] A.D. Martin, Nucl. Phys. B179(1981)33.

[12] M. Iwasaki et al., Phys. Rev. Lett. 78(1997)3067.

[13] A. Gómez Nicola and J.R. Peláez, hep-ph/9909568

[14] T. S. H. Lee, J. A. Oller, E. Oset and A. Ramos, Nucl. Phys A643(1998)402

[15] J. C. Nacher, E. Oset, H. Toki and A. Ramos, Phys. Lett. B455(1999) 55

[16] T. Nakano, Talk at KEK Tanashi Symposium on Physics of Hadrons and Nuclei, Tokyo december 1998

[17] J. C. Nacher, E. Oset, H. Toki and A. Ramos, Phys. Lett. B in print, nucl-th/9902071

[18] J.A. Oller and E. Oset, Phys. Rev. D60 (1999) 074023.

[19] E. Marco, E. Oset and H. Toki, Phys. Rev. C60 (1999) 015202.

[20] M.N. Achasov et al., Phys. Lett. B 440 (1998) 442. R.R. Akhmetshin et al., hep-ph/9907006. M.N. Achasov et al., Phys. Lett. B 438 (1998) 441. R.R. Akhmetshin et al., hep-ph/9907005.

[21] E. Marco, S. Hirenzaki, E. Oset and H. Toki, Phys. Lett. B, in print. 